\title{
Robust Residual- and Recovery-Based A Posteriori Error Estimators for Interface Problems with Flux Jumps
}

\author{
Zhiqiang Cai, Shun Zhang \\ Department of Mathematics, Purdue University, 150 N. University Street, \\ West Lafayette, Indiana 47907-2067
}

Received 13 July 2009; accepted 3 June 2010

Published online in Wiley Online Library (wileyonlinelibrary.com).

DOI 10.1002/num.20629

\begin{abstract}
For elliptic interface problems with flux jumps, this article studies robust residual- and recovery-based a posteriori error estimators for the conforming finite element approximation. The residual estimator is a natural extension of that developed in [Bernardi and Verfürth, Numer Math 85 (2000), 579-608; Petzoldt, Adv Comp Math 16 (2002), 47-75], and the recovery estimator is a nontrivial extension of our method developed in Cai and Zhang, SIAM J Numer Anal 47 (2009) 2132-2156. It is shown theoretically that reliability and efficiency bounds of these error estimators are independent of the jumps provided that the distribution of the coefficients is locally monotone. (C) 2010 Wiley Periodicals, Inc. Numer Methods Partial Differential Eq 000: 000-000, 2010
\end{abstract}

Keywords: a posteriori error estimator; finite element; interface problem

\section{INTRODUCTION}

Let $\Omega$ be a bounded polygonal domain in $\Re^{d}\left(d=2\right.$ or 3 ) with boundary $\partial \Omega=\bar{\Gamma}_{D} \cup \bar{\Gamma}_{N}$ and $\Gamma_{D} \cap \Gamma_{N}=\emptyset$. For simplicity, we assume that $\Gamma_{D}$ is not empty (i.e., mes $\left.\left(\Gamma_{D}\right) \neq 0\right)$. Let $\left\{\Omega_{i}\right\}_{i=1}^{N}$ be a partition of the domain $\Omega$ with $\Omega_{i}$ being an open polygonal subdomain, and let $\mathbf{n}_{i}$ be the outward unit vector normal to the boundary of the domain $\Omega_{i}$. When two subdomains $\Omega_{k}$ and $\Omega_{l}$ share a common boundary, set

$$
\Gamma_{k l}:=\partial \Omega_{k} \cap \partial \Omega_{l}
$$

the interface between $\Omega_{k}$ and $\Omega_{l}$. As $\Gamma_{k l}=\Gamma_{l k}$, we assume that $k<l$ for $\Gamma_{k l}$. The collection of the interior interfaces $\left\{\Gamma_{k l}\right\}$ is denoted by

$$
\mathcal{S}:=\cup_{k<l} \Gamma_{k l}
$$

Correspondence to: Zhiqiang Cai, Department of Mathematics, Purdue University, 150 N. University Street, West Lafayette, IN 47907-2067 (e-mail: zcai@ math.purdue.edu) Contract grant sponsor: The National Science Foundation; contract grant number: DMS-0810855

(c) 2010 Wiley Periodicals, Inc. 
Let $\alpha(x)$ be positive and piecewise constant on polygonal subdomains of $\Omega$ with possible large jumps across subdomain boundaries (interfaces):

$$
\alpha(x)=\alpha_{i}>0 \text { in } \Omega_{i},
$$

for $i=1, \ldots, N$. For a function $v$ defined on the domain $\Omega$, denote by $v_{i}=\left.v\right|_{\Omega_{i}}$ its restriction on $\Omega_{i}$. For any interface $\Gamma_{k l} \in \mathcal{S}$, define the following jumps

$$
\llbracket v \rrbracket_{\Gamma_{k l}}=\left.\left(v_{k}-v_{l}\right)\right|_{\Gamma_{k l}} \quad \text { and } \quad \llbracket \alpha \nabla v \cdot \mathbf{n} \rrbracket_{\Gamma_{k l}}=\left.\left(\alpha_{k} \nabla v_{k} \cdot \mathbf{n}_{k}+\alpha_{l} \nabla v_{l} \cdot \mathbf{n}_{l}\right)\right|_{\Gamma_{k l}} .
$$

In this article, we consider the following interface problem

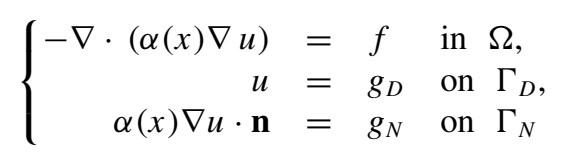

with jump conditions

$$
\llbracket u \rrbracket=0 \quad \text { and } \quad \llbracket \alpha \nabla u \cdot \mathbf{n} \rrbracket=g \quad \text { on } \quad \mathcal{S},
$$

where the symbols $\nabla \cdot$ and $\nabla$ stand for the divergence and gradient operators, respectively, and $f, g, g_{D}$, and $g_{N}$ are given scalar-valued functions in $L^{2}$. For simplicity, we consider the case that the Dirichlet boundary condition $g_{D}$ can be exactly approximated by a continuous piecewise linear function. Problem (1.1)-(1.2) arises in many applications (see, e.g., [1] and the book [2]).

A posteriori error estimations for finite element methods have been extensively studied for the past three decades (see, e.g., books by Verfürth [3], Ainsworth and Oden [4], Babuška and Strouboulis [5], and references therein). For elliptic interface problems without flux jumps, robust a posteriori error estimators have been investigated. For the conforming finite element method, Bernardi and Verfürth [6] and Petzoldt [7] studied a residual-based estimator, and we [8] studied recovery-based estimators that were further extended to mixed and nonconforming finite element methods in [9] and to discontinuous Galerkin finite element methods in [10].

Existing recovery based error estimators, such as Zienkiewicz-Zhu (ZZ) error estimators [11] and Carstensen-Bartels (CB) error estimators [12], recover the gradient/flux in the continuous finite element space. It is well-known that, when the underlying problem is not smooth along the interface, the resulting estimators can significantly over-estimate local error in regions where the approximation is good, and lead to over-refinement. This is shown by Ovall in $[13,14]$ and our numerical results in $[8,9]$. To overcome this difficulty, one often applies the method on each subdomain separately. For reasons why this local approach is not favorable, see [14]. Moreover, those recovery procedures are inexact for problems with Neumann boundary conditions (see e.g., [12]) because they are unable to enforce the Neumann boundary condition. This is particularly true for problems with flux jumps.

The purpose of this article is to develop robust a posteriori error estimators for problem (1.1)-(1.2). In particular, we study an explicit residual estimator for conforming finite element approximation of all orders and a recovery estimator for conforming linear elements. The former is a natural extension of that developed in $[6,7]$ and is analyzed in a similar fashion. The latter is a nontrivial extension of our method developed in [8] because of the flux jumps along interfaces. This is done through a decomposition of the flux: $\sigma=\sigma_{g}+\sigma_{0}$, where $\sigma_{g}$ may be calculated explicitly and $\sigma_{0}$ has continuous normal component across the interfaces and satisfies homogeneous Neumann boundary conditions on $\Gamma_{N}$. Now, the $\sigma_{0}$ may be recovered in a similar fashion 
as those in [8]. The implicit recovery procedure leads to a good approximation to the flux. The resulting recovery estimators based on both the implicit and the explicit recoveries are shown to be robust provided that the distribution of the coefficients are locally monotone. The robustness here means that constants of the reliability and efficiency bound are independent of the jumps of the diffusion coefficients.

This article is organized as follows. Finite element methods and the Clément types of interpolations are described in Sections II. A robust residual-based a posteriori error estimator is introduced and analyzed in Section III. New recovery procedures and the resulting robust recovery-based estimators are introduced and analyzed in Section IV.

\section{FINITE ELEMENT APPROXIMATION AND CLÉMENT-TYPE INTERPOLATION}

We use the standard notations and definitions for the Sobolev spaces $H^{s}(\Omega)^{d}$ and $H^{s}(\partial \Omega)^{d}$ for $s \geq 0$. The standard associated inner products are denoted by $(\cdot, \cdot)_{s, \Omega}$ and $(\cdot, \cdot)_{s, \partial \Omega}$, and their respective norms are denoted by $\|\cdot\|_{s, \Omega}$ and $\|\cdot\|_{s, \partial \Omega}$. (We suppress the superscript $d$ because the dependence on dimension will be clear by context. We also omit the subscript $\Omega$ from the inner product and norm designation when there is no risk of confusion.) For $s=0, H^{s}(\Omega)^{d}$ coincides with $L^{2}(\Omega)^{d}$. In this case, the inner product and norm will be denoted by $\|\cdot\|$ and $(\cdot, \cdot)$, respectively. We will also use the energy norm denoted by

$$
\|v\|=\|v\|_{\Omega}=\left\|\alpha^{1 / 2} \nabla v\right\|_{0, \Omega} .
$$

Let

$$
\begin{gathered}
U:=\left\{v \in H^{1}(\Omega): \llbracket v \rrbracket=0 \text { on } \mathcal{S}\right\} \\
U_{g}:=\left\{v \in U: v=g_{D} \text { on } \Gamma_{D}\right\}, \quad \text { and } \quad U_{0}:=\left\{v \in U: v=0 \text { on } \Gamma_{D}\right\} .
\end{gathered}
$$

Then it is easy to see that the corresponding variational form of Eq. (1.1) with jump conditions (1.2) is to find $u \in U_{g}$ such that

$$
a(u, v)=f(v) \quad \forall v \in U_{0},
$$

where the bilinear and linear forms are defined by

$$
a(u, v)=(\alpha(x) \nabla u, \nabla v)_{\Omega} \quad \text { and } \quad f(v)=(f, v)+\langle g, v\rangle_{\mathcal{S}}+\left\langle g_{N}, v\right\rangle_{\Gamma_{N}},
$$

respectively. Here, $\langle v, w\rangle_{S}=\int_{S} w v d s$ denotes the inner product over $(d-1)$-dimensional manifold $S$.

For the simplicity of presentation, we consider only triangular and tetrahedral elements in the respective two and three dimensions. Let $\mathcal{T}=\{K\}$ be a global conforming finite element partition of the domain $\Omega$, that is, $\mathcal{T}$ has no hanging nodes. Assume that the triangulation $\mathcal{T}$ is regular (see [15]); that is, for all $K \in \mathcal{T}$, there exists a positive constant $\kappa$ such that

$$
h_{K} \leq \kappa \rho_{K},
$$

where $h_{K}$ denotes the diameter of the element $K$ and $\rho_{K}$ the diameter of the largest circle that may be inscribed in $K$. Note that the assumption of the regularity does not exclude highly, locally refined meshes. Furthermore, assume that the interface $\mathcal{S}$ do not cut through any element $K \in \mathcal{T}$. 
Let $P_{k}(K)$ be the space of polynomials of degree $k$ on element $K$. Denote the continuous finite element space of degree $k$ associated with the triangulation $\mathcal{T}$ by

$$
\mathcal{U}_{k}:=\left\{v \in H^{1}(\Omega):\left.v\right|_{K} \in P_{k}(K) \quad \forall K \in \mathcal{T}\right\} \subset U
$$

Let $\mathcal{U}_{k, g}=\left\{v \in \mathcal{U}_{k}: v=g_{D}\right.$ on $\left.\Gamma_{D}\right\}$ and $\mathcal{U}_{k, 0}=\left\{v \in \mathcal{U}_{k}: v=0\right.$ on $\left.\Gamma_{D}\right\}$, then the finite element approximation of (2.1) is to find $u_{\mathcal{T}} \in \mathcal{U}_{k, g}$ such that

$$
a\left(u_{\mathcal{T}}, v\right)=f(v) \quad \forall v \in \mathcal{U}_{k, 0} .
$$

For each element $K \in \mathcal{T}$, denote by $\mathcal{E}_{K}$ the set of its edges/faces. Denote the set of all edges/faces of elements in the triangulation by

$$
\mathcal{E}:=\mathcal{E}_{\Omega} \cup \mathcal{E}_{D} \cup \mathcal{E}_{N} \cup \mathcal{E}_{\mathcal{S}}
$$

where $\mathcal{E}_{D}$ and $\mathcal{E}_{N}$ are the sets of boundary edges/faces, respectively, belonging to $\Gamma_{D}$ and $\Gamma_{N}$; $\mathcal{E}_{\mathcal{S}}$ is the set of interior edges/faces belonging to the interface $\mathcal{S}$; and $\mathcal{E}_{\Omega}$ is the set of all the remaining interior edges/faces. For each $e \in \mathcal{E}$, denote by $\mathbf{n}_{e}$ the fixed unit vector normal to $e$. When $e \in \mathcal{E}_{D} \cup \mathcal{E}_{N}$, assume that $\mathbf{n}_{e}$ is the unit outward vector normal to the boundary $\partial \Omega$. When $e \in \Gamma_{k l} \in \mathcal{E}_{\mathcal{S}}$, let $\mathbf{n}_{e}$ be the unit outward vector normal to the boundary of $\Omega_{k}(k<l)$. For each $e \in \mathcal{E}$, let $\omega_{e}$ denote the union of elements have a common edge/face $e$.

In this article, we assume the following property of the distribution of the coefficients (the Hypothesis 2.7 in [6]) holds.

Assumption on the Distribution of Coefficients. Assume that any two different subdomains $\bar{\Omega}_{i}$ and $\bar{\Omega}_{j}$, which share at least one point, have a connected path passing from $\bar{\Omega}_{i}$ to $\bar{\Omega}_{j}$ through adjacent subdomains such that the diffusion coefficient $\alpha(x)$ is monotone along this path. This assumption is weakened to the quasi-monotonicity in [7].

Lemma 2.1 ([6-8]). For any $v \in U_{0}$, there exists an interpolation operator $I: L^{1}(\Omega) \rightarrow \mathcal{U}_{k, 0}$ such that

$$
\|v-I v\|_{0, K} \leq C h_{K} \alpha_{K}^{-1 / 2}\|v\|_{\Delta_{K}} \quad \text { and } \quad\|\nabla(v-I v)\|_{0, K} \leq C \alpha_{K}^{-1 / 2}\|v\|_{\Delta_{K}}
$$

for all $K \in \mathcal{T}$, where $\Delta_{K}$ is the union of all elements that share at least one vertex with $K$; and that

$$
\|v-I v\|_{0, e} \leq C h_{e}^{1 / 2} \alpha_{e}^{-1 / 2}\|v\|_{\Delta_{e}}
$$

for all $e \in \mathcal{E}$, where $\Delta_{e}$ is the union of all elements that share at least one vertex with $e$, and $\alpha_{e}$ is the arithmetic average of $\alpha$ over $\omega_{e}$ :

$$
\alpha_{e}=\frac{1}{2} \sum_{K \in \omega_{e}} \alpha_{K} \text { for } e \in \mathcal{E}_{\Omega} \cup \mathcal{E}_{\mathcal{S}} \quad \text { and } \quad \alpha_{e}=\alpha_{K} \text { for } e \in \mathcal{E}_{N} \cup \mathcal{E}_{D} .
$$

Remark 2.2. The above lemma is proved in [6-8] for the linear finite element approximation. For higher order finite elements, it can be proved similarly by changing linear nodal basis functions $\phi_{z}$ in [6-8] defined on vertices to higher order Lagrange nodal basis functions defined on nodes of the finite element mesh in the construction of interpolation operator I. 


\section{EXPLICIT RESIDUAL-BASED ERROR ESTIMATOR}

Let $f_{\mathcal{T}}, g_{\mathcal{T}}$, and $g_{N, \mathcal{T}}$ be the $L^{2}$ projections of $f, g$, and $g_{N}$ onto spaces of piecewise polynomials of degree $k-1$ defined on elements of $\mathcal{T}$, interior edges/faces of $\mathcal{E}_{\mathcal{S}}$, and boundary edges/faces of $\mathcal{E}_{N}$, respectively.

Definition 3.1. Define the element residual $R_{K}\left(u_{\mathcal{T}}\right)$ for all $K \in \mathcal{T}$ and the edge jump $J_{e}\left(u_{\mathcal{T}}\right)$ for all $e \in \mathcal{E} \backslash \mathcal{E}_{D}$ as follows

$$
R_{K}\left(u_{\mathcal{T}}\right)=f_{\mathcal{T}}+\nabla \cdot\left(\alpha \nabla u_{\mathcal{T}}\right) \quad \text { and } \quad J_{e}\left(u_{\mathcal{T}}\right)= \begin{cases}-\llbracket \alpha \nabla u_{\mathcal{T}} \cdot \mathbf{n}_{e} \rrbracket & e \in \mathcal{E}_{\Omega}, \\ g_{\mathcal{T}}-\llbracket \alpha \nabla u_{\mathcal{T}} \cdot \mathbf{n}_{e} \rrbracket & e \in \mathcal{E}_{\mathcal{S}}, \\ g_{N, \mathcal{T}}-\alpha \nabla u_{\mathcal{T}} \cdot \mathbf{n}_{e} & e \in \mathcal{E}_{N}\end{cases}
$$

Lemma 3.2. For all $v \in U_{0}$, the residual functional has the following $L^{2}$ representation

$$
\begin{aligned}
(f, v)-a\left(u_{\mathcal{T}}, v\right)= & \sum_{K \in \mathcal{T}}\left(R_{K}\left(u_{\mathcal{T}}\right), v\right)_{K}+\sum_{e \in \mathcal{E} \backslash \mathcal{E}_{D}}\left\langle J_{e}\left(u_{\mathcal{T}}\right), v\right\rangle_{e} \\
& +\left(f-f_{\mathcal{T}}, v\right)+\left\langle g-g_{\mathcal{T}}, v\right\rangle_{\mathcal{S}}+\left\langle g-g_{N, \mathcal{T}}, v\right\rangle_{\Gamma_{N}}
\end{aligned}
$$

Proof. (3.6) is a direct consequence of integration by parts and rearrangement of the summation over edges/faces.

Definition 3.3. Define the residual based error estimator and its local error indicator on $K$ as follows

$$
\begin{aligned}
\eta_{R} & =\left(\sum_{K \in \mathcal{T}} \eta_{K}^{2}\right)^{1 / 2} \\
\eta_{K} & =\left(\frac{h_{K}^{2}}{\alpha_{K}}\left\|R_{K}\left(u_{\mathcal{T}}\right)\right\|_{0, K}^{2}+\sum_{e \in \mathcal{E}_{K} \cap\left(\mathcal{E}_{\Omega} \cup \mathcal{E}_{\mathcal{S}}\right)} \frac{h_{e}}{2 \alpha_{e}}\left\|J_{e}\left(u_{\mathcal{T}}\right)\right\|_{0, e}^{2}+\sum_{e \in \mathcal{E}_{K} \cap \mathcal{E}_{N}} \frac{h_{e}}{\alpha_{e}}\left\|J_{e}\left(u_{\mathcal{T}}\right)\right\|_{0, e}^{2}\right)^{1 / 2}
\end{aligned}
$$

Definition 3.4. For the linear finite element approximation, define the edge error estimator and its local error indicator on e as follows

$$
\eta_{E}=\left(\sum_{e \in \mathcal{E} \backslash \mathcal{E}_{D}} \eta_{e}^{2}\right)^{1 / 2} \text { and } \eta_{e}=h_{e}^{1 / 2} \alpha_{e}^{-1 / 2}\left\|J_{e}\left(u_{\mathcal{T}}\right)\right\|_{0, e} .
$$

Denote by $\mathcal{N}$ and $\mathcal{N}_{K}$ the sets of all vertcies of the triangulation $\mathcal{T}$ and of the element $K \in \mathcal{T}$, respectively. For any $z \in \mathcal{N}$, denote by $\phi_{z}$ the linear nodal basis function, let $\omega_{z}=\operatorname{supp}\left(\phi_{z}\right)$. For a given function $v$, define its weighted average over $\omega_{z}$ by

$$
f_{\omega_{z}} v d x=\frac{\int_{\omega_{z}} v \phi_{z} d x}{\int_{\omega_{z}} \phi_{z} d x}
$$


Let

$$
\begin{aligned}
H_{f} & =\left(\sum_{K \in \mathcal{T}} H_{f, K}^{2}\right)^{1 / 2} \text { with } H_{f, K}=\frac{h_{K}}{\sqrt{\alpha_{K}}}\left\|f-f_{\mathcal{T}}\right\|_{0, K} \quad \forall K \in \mathcal{T}, \\
H_{g} & =\left(\sum_{e \in \mathcal{E}_{\mathcal{S}}} H_{g, e}^{2}\right)^{1 / 2} \text { with } H_{g, e}=\sqrt{\frac{h_{e}}{\alpha_{e}}}\left\|g-g_{\mathcal{T}}\right\|_{0, e} \quad \forall e \in \mathcal{E}_{\mathcal{S}}, \\
H_{g_{N}} & =\left(\sum_{e \in \mathcal{E}_{N}} H_{g_{N}, e}^{2}\right)^{1 / 2} \quad \text { with } H_{g_{N}, e}=\sqrt{\frac{h_{e}}{\alpha_{e}}}\left\|g_{N}-g_{N, \mathcal{T}}\right\|_{0, e} \quad \forall e \in \mathcal{E}_{N},
\end{aligned}
$$

and for linear finite approximations, let

$$
\hat{H}_{f}=\left(\sum_{z \in \mathcal{N} \cap\left(\mathcal{S} \cup \Gamma_{D}\right)} \sum_{K \subset \omega_{z}} \frac{h_{K}^{2}}{\alpha_{K}}\|f\|_{0, K}^{2}+\sum_{z \in \mathcal{N} \backslash\left(\mathcal{S} \cup \Gamma_{D}\right)} \sum_{K \subset \omega_{z}} \frac{h_{K}^{2}}{\alpha_{K}}\left\|f-f_{\omega_{z}} f d x\right\|_{0, K}^{2}\right)^{1 / 2} .
$$

Remark 3.5. For the linear finite element approximation, the second term in $\hat{H}_{f}$ is of higher order than $\eta_{E}$ for $f \in L^{2}(\Omega)$ and so is the first term for $f \in L^{p}(\Omega)$ with $p>2$ (see [16]).

Lemma 3.6. For any $v \in U_{0}$, there exists a positive constant $C$ independent of the ratio $\alpha_{\max } / \alpha_{\min }$ such that

$$
\begin{aligned}
& \left|\left(f-f_{\mathcal{T}}, v-I v\right)\right| \leq C H_{f}\|v\|, \\
& \left|\left\langle g-g_{\mathcal{T}}, v-I v\right\rangle_{\mathcal{S}}\right| \leq C H_{g}\|v\| \quad \text { and } \quad\left|\left\langle g_{N}-g_{N_{\mathcal{T}}}, v-I v\right\rangle_{\Gamma_{N}}\right| \leq C H_{g_{N}}\|v\| .
\end{aligned}
$$

and for the linear finite element approximation,

$$
|(f, v-I v)| \leq C \hat{H}_{f}\|v\|
$$

Proof. The inequality in (3.12) is established in [8]. The inequality in (3.10) follows from the Cauchy-Schwarz inequality and (2.3) that

$$
\left(f-f_{\mathcal{T}}, v-I v\right) \leq\left(\sum_{K \in \mathcal{T}} \frac{h_{K}^{2}}{\alpha_{K}}\left\|f-f_{\mathcal{T}}\right\|_{0, K}^{2}\right)^{1 / 2}\left(\sum_{K \in \mathcal{T}} \frac{\alpha_{K}}{h_{K}^{2}}\|v-I v\|_{0, K}^{2}\right)^{1 / 2} \leq C H_{f}\|v\| .
$$

(3.11) may be proved in the same fashion.

Theorem 3.7 (Reliability). Denote the true error of the solution by $e=u-u_{\mathcal{T}}$. The error estimator $\eta_{R}$ satisfies the following global reliability bound:

$$
\|e\|_{\Omega} \leq C\left(\eta_{R}+H_{f}+H_{g}+H_{g_{N}}\right) .
$$

For the linear finite element approximation, the edge error estimator $\eta_{E}$ satisfies the following global reliability bound:

$$
\|e\|_{\Omega} \leq C\left(\eta_{E}+\hat{H}_{f}+H_{g}+H_{g_{N}}\right) .
$$

The constants in both bounds are independent of the ratio $\alpha_{\max } / \alpha_{\min }$.

Numerical Methods for Partial Differential Equations DOI 10.1002/num 
Proof. It follows from the orthogonality property of the finite element solution, Lemma 4.2, (2.3), (2.4), and Lemma 3.6 that

$$
\begin{aligned}
\|e\|_{\Omega}^{2}= & a(e, e-I e) \\
= & \sum_{K \in \mathcal{T}}\left(R_{K}\left(u_{\mathcal{T}}\right), e-I e\right)_{K}+\sum_{e \in \mathcal{E} \backslash \mathcal{E}_{D}}\left\langle J_{e}\left(u_{\mathcal{T}}\right), e-I e\right\rangle_{e} \\
& +\left(f-f_{\mathcal{T}}, e-I e\right)+\left\langle g-g_{\mathcal{T}}, e-I e\right\rangle_{\mathcal{S}}+\left\langle g_{N}-g_{N, \mathcal{T}}, e-I e\right\rangle_{\Gamma_{N}} \\
\leq & C \sum_{K \in \mathcal{T}} \frac{h_{K}}{\sqrt{\alpha_{K}}}\left\|R_{K}\left(u_{\mathcal{T}}\right)\right\|_{0, K}\|e\|_{\Delta_{K}}+C \sum_{e \in \mathcal{E} \backslash \mathcal{E}_{D}} \sqrt{\frac{h_{e}}{\alpha_{e}}}\left\|J_{e}\left(u_{\mathcal{T}}\right)\right\|_{0, e}\|e\|_{\Delta_{e}} \\
& +C\left(H_{f}+H_{g}+H_{g_{N}}\right)\|e\|_{\Omega} .
\end{aligned}
$$

Thus,

$$
\|e\| \leq C\left(\eta_{R}+H_{f}+H_{g}+H_{g_{N}}\right) .
$$

For the linear finite element approximation, since $\nabla \cdot\left(\alpha \nabla u_{\mathcal{T}}\right)=0$ on each element $K \in \mathcal{T}$, we have

$$
\begin{aligned}
\|e\|_{\Omega}^{2} & =a(e, e-I e) \\
& =(f, e-I e)+\sum_{e \in \mathcal{E}}\left\langle J_{e}\left(u_{\mathcal{T}}\right), e-I e\right\rangle_{e}+\left\langle g-g_{\mathcal{T}}, e-I e\right\rangle_{\mathcal{S}}+\left\langle g_{N}-g_{N, \mathcal{T}}, e-I e\right\rangle_{\Gamma_{N}} \\
& \leq C \sum_{e \in \mathcal{E} \backslash \mathcal{E}_{D}} h_{e}^{1 / 2} \alpha_{e}^{-1 / 2}\left\|J_{e}\left(u_{\mathcal{T}}\right)\right\|_{0, e}\|e\|_{\Delta_{e}}+C\left(\hat{H}_{f}+H_{g}+H_{g_{N}}\right)\|e\|_{\Omega},
\end{aligned}
$$

which implies (3.14). This completes the proof of the theorem.

Lemma 3.8. There exists a bubble function $\psi_{K} \in H_{0}^{1}(K)$ such that for any $v \in P_{k}(K)$,

$$
C\|v\|_{0, K} \leq\left\|v \psi_{K}\right\|_{0, K} \leq\left\|v \psi_{K}^{1 / 2}\right\|_{0, K} \leq\|v\|_{0, K},
$$

and a bubble function $\psi_{e} \in H_{0}^{1}\left(\omega_{e}\right)$ such that for any $v \in P_{k}(K)$ and any $K \in \omega_{e}$,

$$
C\|v\|_{0, e} \leq\left\|v \psi_{e}\right\|_{0, e} \leq\left\|v \psi_{e}^{1 / 2}\right\|_{0, e} \leq\|v\|_{0, e} .
$$

Proof. The lemma is proved in [3].

Lemma 3.9 (Efficiency). Without the assumption on the distribution of the coefficients $\alpha$, there exists a positive constant $C$ independent of $\alpha_{\max } / \alpha_{\min }, h_{K}$, and $h_{e}$ such that

$$
h_{K}^{2} \alpha_{K}^{-1}\left\|R_{K}\left(u_{\mathcal{T}}\right)\right\|_{0, K}^{2} \leq C\left(\|e\|_{K}^{2}+H_{f, K}^{2}\right)
$$

and that

$$
h_{e} \alpha_{e}^{-1}\left\|J_{e}\left(u_{\mathcal{T}}\right)\right\|_{0, e}^{2} \leq C\left(\|e\|_{\omega_{e}}^{2}+\sum_{K \in \mathcal{T} \cap \omega_{e}} H_{f, K}^{2}+H_{g, e}^{2}+H_{g_{N}, e}^{2}\right)
$$


Proof. (3.17) may be proved by choosing the test function $v=R_{K}\left(u_{\mathcal{T}}\right) \psi_{K}$ in (3.6) and following the standard argument in [3]. Similarly, (3.18) may be proved by choosing the test function $v=J_{e}\left(u_{\mathcal{T}}\right) \psi_{e}$ in (3.6)

\section{FLUX RECOVERY AND ERROR ESTIMATOR}

In this section, we assume that the solution $u \in H^{t}\left(\Omega_{i}\right), t>3 / 2$, for $i=1, \ldots, N$.

The flux is an important physical quantity defined by

$$
\sigma=-\alpha(x) \nabla u \text { in } \Omega .
$$

For the interface problem in (1.1) with the jump conditions in (1.2), as $f \in L^{2}(\Omega)$, it is then known that

$$
\boldsymbol{\sigma} \in H\left(\operatorname{div} ; \Omega_{i}\right):=\left\{\boldsymbol{\tau} \in L^{2}\left(\Omega_{i}\right)^{d}: \nabla \cdot \boldsymbol{\tau} \in L^{2}\left(\Omega_{i}\right)\right\}
$$

for $i=1, \ldots, N$. The $H\left(\operatorname{div} ; \Omega_{i}\right)$ is a Hilbert space under the norm

$$
\|\boldsymbol{\tau}\|_{H\left(\mathrm{div} ; \Omega_{i}\right)}:=\left(\|\boldsymbol{\tau}\|_{0, \Omega_{i}}^{2}+\|\nabla \cdot \boldsymbol{\tau}\|_{0, \Omega_{i}}^{2}\right)^{\frac{1}{2}} .
$$

Notice under the regularity assumption that $\left.u\right|_{\Omega_{i}} \in H^{t}\left(\Omega_{i}\right), t>3 / 2$, by the trace theorem (see e.g. [17]), $\left.\boldsymbol{\sigma} \cdot \mathbf{n}\right|_{\partial \Omega_{i}}=-\left.\alpha \nabla u \cdot \mathbf{n}\right|_{\partial \Omega_{i}} \in L^{2}\left(\partial \Omega_{i}\right)$.

Define the corresponding space on the whole domain $\Omega$ by

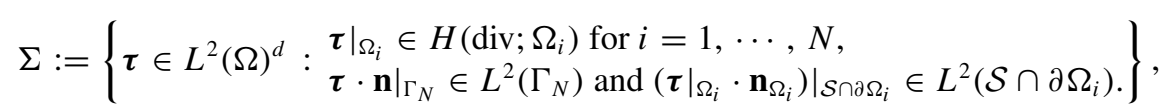

which is equipped with the broken $H$ (div) norm

$$
\|\boldsymbol{\tau}\|_{\Sigma}=\left(\sum_{i=1}^{N}\|\boldsymbol{\tau}\|_{H\left(\mathrm{div} ; \Omega_{i}\right)}^{2}\right)^{1 / 2}
$$

Denote by

$$
\Sigma_{g}:=\left\{\boldsymbol{\tau} \in \Sigma: \boldsymbol{\tau} \cdot \mathbf{n}=-g_{N} \text { on } \Gamma_{N} \text { and } \llbracket \boldsymbol{\tau} \cdot \mathbf{n} \rrbracket=-g \text { on } \mathcal{S}\right\},
$$

and let

$$
\Sigma_{0}:=\left\{\boldsymbol{\tau} \in H(\operatorname{div} ; \Omega): \boldsymbol{\tau} \cdot \mathbf{n}=0 \text { on } \Gamma_{N}\right\} .
$$

The flux satisfies the following problem: find $\sigma \in \Sigma_{g}$ such that

$$
\left(\alpha^{-1} \boldsymbol{\sigma}, \boldsymbol{\tau}\right)=-(\nabla u, \boldsymbol{\tau}) \quad \forall \boldsymbol{\tau} \in \Sigma_{0} .
$$

Since $g_{N}$ and $g$ are $L^{2}$ functions on $\Gamma_{N}$ and $\mathcal{S}$, respectively, there exists a function $\sigma_{g} \in \Sigma$ such that $\boldsymbol{\sigma}_{g} \cdot \mathbf{n}=-g_{N}$ on $\Gamma_{N}$ and that $\llbracket \boldsymbol{\sigma}_{g} \cdot \mathbf{n} \rrbracket=-g$ on $\mathcal{S}$. Then the flux has the following decomposition

$$
\boldsymbol{\sigma}=\sigma_{g}+\sigma_{0}
$$


where $\sigma_{0}=\sigma-\sigma_{g} \in \Sigma_{0}$ satisfies

$$
\left(\alpha^{-1} \boldsymbol{\sigma}_{0}, \boldsymbol{\tau}\right)=-(\nabla u, \boldsymbol{\tau})-\left(\alpha^{-1} \boldsymbol{\sigma}_{g}, \boldsymbol{\tau}\right) \quad \forall \boldsymbol{\tau} \in \Sigma_{0} .
$$

Note that this decomposition is not unique.

\section{A. Flux Recovery}

The recovery procedure introduced in this article is based on the $H$ (div) conforming finite element approximation to the variational problem in (4.3). There are several families of the $H$ (div) conforming finite element spaces (see, e.g., [18]), but we consider only Raviart-Thomas elements [19] for simplicity.

Denote the local lowest order Raviart-Thomas space on a element $K \in \mathcal{T}$ by

$$
R T_{0}(K)=P_{0}(K)^{d}+\mathbf{x} P_{0}(K), \quad \mathbf{x}=\left(x_{1}, \ldots, x_{d}\right) .
$$

Then piecewise $H$ (div) conforming Raviart-Thomas space with respect to the subdomains $\left\{\Omega_{i}\right\}$ are defined as follows

$$
\mathcal{V}=\left\{\boldsymbol{\tau} \in \Sigma:\left.\tau\right|_{K} \in R T_{0}(K) \quad \forall K \in \mathcal{T}\right\}
$$

Implicit Approximation. Let

$$
\mathcal{V}_{g}:=\left\{\boldsymbol{\tau} \in \mathcal{V}: \boldsymbol{\tau} \cdot \mathbf{n}=-g_{N, \mathcal{T}} \text { on } \Gamma_{N} \text { and } \llbracket \boldsymbol{\tau} \cdot \mathbf{n} \rrbracket=-g_{\mathcal{T}} \text { on } \mathcal{S}\right\},
$$

and

$$
\mathcal{V}_{0}:=\mathcal{V} \cap \Sigma_{0}
$$

Define $\sigma_{g, \mathcal{T}} \in \mathcal{V}_{g}$ by setting its normal components: single value $\sigma_{g, e}$ for all $e \in \mathcal{E}_{\Omega} \cup \mathcal{E}_{D} \cup \mathcal{E}_{N}$ and multiple values $\sigma_{g, e}^{ \pm}$for all $e \in \mathcal{E}_{\mathcal{S}}$ as follows

$$
\sigma_{g, e}=\left\{\begin{array}{ll}
-g_{N, \mathcal{T}} & \text { in } \mathcal{E}_{N}, \\
0 & \text { in } \mathcal{E}_{\Omega} \cup \mathcal{E}_{D}
\end{array} \quad \text { and } \quad \sigma_{g, e}^{+}=-g_{\mathcal{T}}, \quad \sigma_{g, e}^{-}=0 \quad \text { in } \mathcal{E}_{\mathcal{S}}\right.
$$

Let $\bar{u}_{\mathcal{T}} \in \mathcal{U}_{1}$ be a linear finite element approximation of the exact solution $u$ of (2.1), then the recovered flux is defined by

$$
\sigma_{\mathcal{T}}=\sigma_{0, \mathcal{T}}+\sigma_{g, \mathcal{T}}
$$

where $\sigma_{0, \mathcal{T}} \in \mathcal{V}_{0}$ satisfies

$$
\left(\alpha^{-1} \boldsymbol{\sigma}_{0, \mathcal{T}}, \boldsymbol{\tau}\right)=-\left(\nabla \bar{u}_{\mathcal{T}}, \boldsymbol{\tau}\right)-\left(\alpha^{-1} \boldsymbol{\sigma}_{g, \mathcal{T}}, \boldsymbol{\tau}\right) \quad \forall \boldsymbol{\tau} \in \mathcal{V}_{0}
$$

First, we estimate difference between $\boldsymbol{\sigma}_{g}$ and $\boldsymbol{\sigma}_{g, \mathcal{T}}$.

Lemma 4.1. There exists a function $\sigma_{g} \in \Sigma_{g}$ such that

$$
\left\|\alpha^{-1 / 2}\left(\boldsymbol{\sigma}_{g}-\sigma_{g, \mathcal{T}}\right)\right\|_{0, \Omega} \leq C\left(H_{g}^{2}+H_{g_{N}}^{2}\right)^{1 / 2}
$$


Proof. Let $\sigma_{g}=\sigma_{g, \mathcal{T}}-\alpha \nabla v$, where the function $v$ satisfies the following problem:

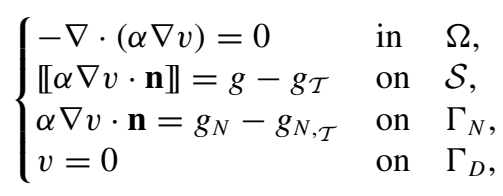

whose variational formulation is to find $v \in U_{0}$, such that

$$
a(v, w)=\left\langle g-g_{\mathcal{T}}, w\right\rangle_{\mathcal{S}}+\left\langle g_{N}-g_{N, \mathcal{T}}, w\right\rangle_{\Gamma_{N}} \quad \forall w \in U_{0}
$$

Since $g_{\mathcal{T}}$ and $g_{N, \mathcal{T}}$ are the $L^{2}$ projections of $g$ and $g_{N}$ onto spaces of piecewise constants defined on interior edges/faces of $\mathcal{E}_{\mathcal{S}}$ and boundary edges/faces of $\mathcal{E}_{N}$, respectively, we have

$$
\left\langle g-g_{\mathcal{T}}, c\right\rangle_{e}=0 \quad \forall e \in \mathcal{E}_{\mathcal{S}} \quad \text { and } \quad\left\langle g_{N}-g_{N, \mathcal{T}}, c\right\rangle_{e}=0 \quad \forall e \in \mathcal{E}_{N}
$$

for any constant $c \in \Re$. Denote by $\bar{v}_{e}$ be the average of $v$ on the edge $e$. Also denote by $K_{\max , e}$ be the element has a bigger $\alpha_{K}$ in $\omega_{e}$. If there are two $K$ 's that have the same $\alpha_{K}$ in $\omega_{e}$, then pick an arbitrary one and denote it as $K_{\max , e}$. Choosing $w=v$ in (4.7), then it follows from (4.8), the Cauchy-Schwarz inequality, trace inequality, and approximation theory that

$$
\begin{aligned}
\left\|\alpha^{1 / 2} \nabla v\right\|_{0}^{2} & =\left\langle g-g_{\mathcal{T}}, v\right\rangle_{\mathcal{S}}+\left\langle g_{N}-g_{N, \mathcal{T}}, v\right\rangle_{\Gamma_{N}} \\
& =\sum_{e \in \mathcal{E}_{\mathcal{S}}}\left\langle g-g_{\mathcal{T}}, v-\bar{v}_{e}\right\rangle_{e}+\sum_{e \in \mathcal{E}_{N}}\left\langle g_{N}-g_{N, \mathcal{T}}, v-\bar{v}_{e}\right\rangle_{e} \\
& \leq \sum_{e \in \mathcal{E}_{\mathcal{S}}} H_{g, e} h_{e}^{-1 / 2} \alpha_{e}^{1 / 2}\left\|v-\bar{v}_{e}\right\|_{0, e}+\sum_{e \in \mathcal{E}_{N}} H_{g_{N}, e} h_{e}^{-1 / 2} \alpha_{e}^{1 / 2}\left\|v-\bar{v}_{e}\right\|_{0, e} \\
& \leq C \sum_{e \in \mathcal{E}_{\mathcal{S}}} H_{g, e}\left\|\alpha^{\frac{1}{2}} \nabla v\right\|_{0, K_{\max , e}}+C \sum_{e \in \mathcal{E}_{N}} H_{g_{N}, e}\left\|\alpha^{\frac{1}{2}} \nabla v\right\|_{0, K_{\max , e}} \\
& \leq C\left(H_{g}^{2}+H_{g_{N}}^{2}\right)^{1 / 2}\left\|\alpha^{1 / 2} \nabla v\right\|_{0, \Omega},
\end{aligned}
$$

which implies

$$
\left\|\alpha^{-1 / 2}\left(\sigma_{g}-\sigma_{g, \mathcal{T}}\right)\right\|_{0, \Omega}=\left\|\alpha^{1 / 2} \nabla v\right\|_{0, \Omega} \leq C\left(H_{g}^{2}+H_{g_{N}}^{2}\right)^{1 / 2} .
$$

This completes the proof of the lemma.

Theorem 4.2. Let $u$ be the solution of (2.1). Then there exists a positive constant $C$ independent of the ratio $\alpha_{\max } / \alpha_{\min }$ such that

$$
\left\|\alpha^{-1 / 2}\left(\boldsymbol{\sigma}-\boldsymbol{\sigma}_{\mathcal{T}}\right)\right\|_{0, \Omega} \leq C\left(\inf _{\boldsymbol{\tau} \in \mathcal{V}_{0}}\left\|\alpha^{-1 / 2}\left(\sigma_{0}-\boldsymbol{\tau}\right)\right\|_{0, \Omega}+\left\|u-\bar{u}_{\mathcal{T}}\right\|_{\Omega}+\left(H_{g}^{2}+H_{g_{N}}^{2}\right)^{1 / 2}\right)
$$

Proof. Let $\sigma_{g}$ be chosen as in Lemma 4.1, then

$$
\sigma-\sigma_{\mathcal{T}}=\left(\sigma_{g}-\sigma_{g, \mathcal{T}}\right)+\left(\sigma_{0}-\sigma_{0, \mathcal{T}}\right)
$$


which, together with the triangle inequality, implies

$$
\left\|\alpha^{-1 / 2}\left(\boldsymbol{\sigma}-\boldsymbol{\sigma}_{\mathcal{T}}\right)\right\|_{0, \Omega} \leq\left\|\alpha^{-1 / 2}\left(\boldsymbol{\sigma}_{g}-\boldsymbol{\sigma}_{g, \mathcal{T}}\right)\right\|_{0, \Omega}+\left\|\alpha^{-1 / 2}\left(\boldsymbol{\sigma}_{0}-\boldsymbol{\sigma}_{0, \mathcal{T}}\right)\right\|_{0, \Omega}
$$

The difference of Eqs. (4.3) and (4.5) gives

$$
\left(\alpha^{-1}\left(\boldsymbol{\sigma}_{0}-\boldsymbol{\sigma}_{0, \mathcal{T}}\right), \boldsymbol{\tau}\right)=-\left(\nabla\left(u-\bar{u}_{\mathcal{T}}\right), \boldsymbol{\tau}\right)-\left(\alpha^{-1}\left(\boldsymbol{\sigma}_{g}-\boldsymbol{\sigma}_{g, \mathcal{T}}\right), \boldsymbol{\tau}\right) \quad \forall \boldsymbol{\tau} \in \mathcal{V}_{0},
$$

Combining with the Cauchy-Schwarz and triangle inequalities yields

$$
\begin{aligned}
\left\|\alpha^{-1 / 2}\left(\boldsymbol{\sigma}_{0}-\boldsymbol{\sigma}_{0, \mathcal{T}}\right)\right\|_{0, \Omega}^{2} & \\
= & \left(\alpha^{-1}\left(\boldsymbol{\sigma}_{0}-\boldsymbol{\sigma}_{0, \mathcal{T}}\right), \boldsymbol{\sigma}_{0}-\boldsymbol{\tau}\right)-\left(\nabla\left(u-\bar{u}_{\mathcal{T}}\right), \boldsymbol{\tau}-\boldsymbol{\sigma}_{0, \mathcal{T}}\right)-\left(\alpha^{-1}\left(\boldsymbol{\sigma}_{g}-\boldsymbol{\sigma}_{g, \mathcal{T}}\right), \boldsymbol{\tau}-\boldsymbol{\sigma}_{0, \mathcal{T}}\right) \\
\leq & \left\|\alpha^{-1 / 2}\left(\boldsymbol{\sigma}_{0}-\boldsymbol{\sigma}_{0, \mathcal{T}}\right)\right\|_{0, \Omega}\left\|\alpha^{-1 / 2}\left(\boldsymbol{\sigma}_{0}-\boldsymbol{\tau}\right)\right\|_{0, \Omega} \\
& \quad+\left(\left\|u-\bar{u}_{\mathcal{T}}\right\|_{\Omega}+\left\|\alpha^{-1 / 2}\left(\boldsymbol{\sigma}_{g}-\boldsymbol{\sigma}_{g, \mathcal{T}}\right)\right\|_{0, \Omega}\right)\left(\left\|\alpha^{-1 / 2}\left(\boldsymbol{\tau}-\boldsymbol{\sigma}_{0}\right)\right\|_{0, \Omega}+\left\|\alpha^{-1 / 2}\left(\boldsymbol{\sigma}_{0}-\boldsymbol{\sigma}_{0, \mathcal{T}}\right)\right\|_{0, \Omega}\right) .
\end{aligned}
$$

Hence,

$$
\left\|\alpha^{-\frac{1}{2}}\left(\boldsymbol{\sigma}_{0}-\boldsymbol{\sigma}_{0, \mathcal{T}}\right)\right\|_{0, \Omega} \leq C\left(\inf _{\boldsymbol{\tau} \in \mathcal{V}_{0}}\left\|\alpha^{-1 / 2}\left(\boldsymbol{\sigma}_{0}-\boldsymbol{\tau}\right)\right\|_{0, \Omega}+\left\|u-\bar{u}_{\mathcal{T}}\right\|_{\Omega}+\left\|\alpha^{-\frac{1}{2}}\left(\boldsymbol{\sigma}_{g}-\boldsymbol{\sigma}_{g, \mathcal{T}}\right)\right\|_{0, \Omega}\right) .
$$

Now, the theorem is a direct consequence of (4.9) and Lemma 4.1.

Explicit Approximations. The nodal basis function $\phi_{e}$ of $R T_{0}$ corresponding to $e \in \mathcal{E}$ is characterized by

$$
\left.\boldsymbol{\phi}_{e} \cdot \mathbf{n}_{e^{\prime}}\right|_{e^{\prime}}=\delta_{e e^{\prime}} \quad \forall e, e^{\prime} \in \mathcal{E},
$$

where $\delta_{e e^{\prime}}$ is the Kronecker delta. For each edge/face $e \in \mathcal{E}_{\Omega} \cup \mathcal{E}_{\mathcal{S}}$, let $K_{e}^{+}$and $K_{e}^{-}$be the two elements sharing the common edge/face $e$ such that the unit outward normal vector of $K_{e}^{+}$coincides with $\mathbf{n}_{e}$. For each edge/face $e \in \mathcal{E}_{\mathcal{S}}$ and $e \subset \Gamma_{k l}$ with $k<l$, we always choose $K_{e}^{+} \subset \Omega_{k}$ and $K_{e}^{-} \subset \Omega_{l}$.

Let $\boldsymbol{\tau}=-\alpha(x) \nabla \bar{u}_{\mathcal{T}}$. For any interior edge/face $e \in \mathcal{E}_{\Omega} \cup \mathcal{E}_{\mathcal{S}}$, denote restrictions of $\boldsymbol{\phi}_{e}$ and $\boldsymbol{\tau}$ on $K_{e}^{+}$and $K_{e}^{-}$by

$$
\boldsymbol{\phi}_{e}^{+}=\left.\boldsymbol{\phi}_{e}\right|_{K_{e}^{+}}, \quad \boldsymbol{\phi}_{e}^{-}=\left.\boldsymbol{\phi}_{e}\right|_{K_{e}^{-}}, \quad \boldsymbol{\tau}^{+}=\left.\boldsymbol{\tau}\right|_{K_{e}^{+}}, \quad \text { and } \quad \boldsymbol{\tau}^{+}=\left.\boldsymbol{\tau}\right|_{K_{e}^{+}} .
$$

Define an approximation, $\hat{\boldsymbol{\sigma}}_{\mathcal{T}}\left(\bar{u}_{\mathcal{T}}\right)$, of $\boldsymbol{\tau}$ in $R T_{0}$ by

$$
\hat{\boldsymbol{\sigma}}_{\mathcal{T}}\left(\bar{u}_{\mathcal{T}}\right)=\sum_{e \in \mathcal{E}_{\Omega} \cup \mathcal{E}_{D} \cup \mathcal{E}_{N}} \hat{\sigma}_{e} \boldsymbol{\phi}_{e}(\mathbf{x})+\sum_{e \in \mathcal{E}_{\mathcal{S}}}\left(\hat{\sigma}_{e}^{+} \boldsymbol{\phi}_{e}^{+}(\mathbf{x})+\hat{\sigma}_{e}^{-} \boldsymbol{\phi}_{e}^{-}(\mathbf{x})\right),
$$

where $\hat{\sigma}_{e}$ on $e \in \mathcal{E}_{\Omega} \cup \mathcal{E}_{D} \cup \mathcal{E}_{N}$ is the normal component of $\hat{\boldsymbol{\sigma}}_{\mathcal{T}}$ defined by

$$
\hat{\sigma}_{e}:= \begin{cases}\left.\gamma_{e}\left(\boldsymbol{\tau}_{e}^{+} \cdot \mathbf{n}_{e}\right)\right|_{e}+\left.\left(1-\gamma_{e}\right)\left(\boldsymbol{\tau}_{e}^{-} \cdot \mathbf{n}_{e}\right)\right|_{e} & \text { for } e \in \mathcal{E}_{\Omega}, \\ \left.\boldsymbol{\tau}\right|_{e} \cdot \mathbf{n}_{e} & \text { for } e \in \mathcal{E}_{D}, \\ -g_{N,\left.\mathcal{T}\right|_{e}} & \text { for } e \in \mathcal{E}_{N}\end{cases}
$$


are the normal components of $\hat{\boldsymbol{\sigma}}_{\mathcal{T}}$ defined by

$$
\begin{aligned}
\hat{\sigma}_{e}^{+} & =\left.\gamma_{e}\left(\boldsymbol{\tau}_{e}^{+} \cdot \mathbf{n}_{e}\right)\right|_{e}-\left.\left(1-\gamma_{e}\right) g_{h}\right|_{e}+\left.\left(1-\gamma_{e}\right)\left(\boldsymbol{\tau}_{e}^{-} \cdot \mathbf{n}_{e}\right)\right|_{e} \\
\text { and } \quad \hat{\sigma}_{e}^{-} & =\left.\gamma_{e}\left(\boldsymbol{\tau}_{e}^{+} \cdot \mathbf{n}_{e}\right)\right|_{e}+\left.\gamma_{e} g_{h}\right|_{e}+\left.\left(1-\gamma_{e}\right)\left(\boldsymbol{\tau}_{e}^{-} \cdot \mathbf{n}_{e}\right)\right|_{e}
\end{aligned}
$$

for some constant $\gamma_{e} \in[0,1]$. In this article, we choose

$$
\gamma_{e}=\frac{\alpha_{K_{e}^{-}}}{\alpha_{K_{e}^{+}}+\alpha_{K_{e}^{-}}} \quad \text { or } \quad \frac{\sqrt{\alpha_{K_{e}^{-}}}}{\sqrt{\alpha_{K_{e}^{+}}}+\sqrt{\alpha_{K_{e}^{-}}}}
$$

to ensure that the efficiency constant on the corresponding a posteriori error estimator is independent of the ratio $\alpha_{\max } / \alpha_{\min }$ (see Theorem 5.4). Notice, with either special choice of $\gamma_{e}$, the following inequality holds,

$$
\frac{\left(1-\gamma_{e}\right)^{2}}{\alpha_{K_{e}^{+}}} \leq \frac{1}{\alpha_{K_{e}^{+}}+\alpha_{K_{e}^{-}}} .
$$

Note that the approximation, $\hat{\boldsymbol{\sigma}}_{\mathcal{T}}\left(\bar{u}_{\mathcal{T}}\right)$, defined in (4.11) has continuous normal component across $e \in \mathcal{E}_{\Omega}$ and preserves the flux jump on $e \in \mathcal{E}_{\mathcal{S}}$ :

$$
\hat{\sigma}_{e}^{+}-\hat{\sigma}_{e}^{-}=-\left.g_{\mathcal{T}}\right|_{e}
$$

\section{B. Recovery-Based Error Estimators}

Let $\sigma_{\mathcal{T}}$ be the recovered flux defined in (4.4), define the following local a posteriori error indicator by

$$
\eta_{\mathcal{V}, K}=\left\|\alpha^{-1 / 2} \boldsymbol{\sigma}_{\mathcal{T}}+\alpha^{1 / 2} \nabla \bar{u}_{\mathcal{T}}\right\|_{0, K}
$$

for any element $K \in \mathcal{T}$. Then the corresponding global a posteriori error estimator is

$$
\eta_{\mathcal{V}}=\left(\sum_{K \in \mathcal{T}}\left(\eta_{\mathcal{V}, K}\right)^{2}\right)^{1 / 2}=\left\|\alpha^{-1 / 2} \sigma_{\mathcal{T}}+\alpha^{1 / 2} \nabla \bar{u}_{\mathcal{T}}\right\|_{0, \Omega}
$$

From the construction of $\sigma_{\mathcal{T}}$, it is easy to see that every function $\tau \in \mathcal{V}_{g}$ can be written as the sum of $\boldsymbol{\sigma}_{g, \mathcal{T}}$ and $\boldsymbol{\tau}_{0, \mathcal{T}}$ with $\boldsymbol{\tau}_{0, \mathcal{T}} \in \mathcal{V}_{0}$. For fixed $\boldsymbol{\sigma}_{g, \mathcal{T}}$, this construction is unique. Thus,

$$
\eta_{\mathcal{V}}=\min _{\boldsymbol{\tau} \in \mathcal{V}_{0}}\left\|\alpha^{-1 / 2}\left(\boldsymbol{\tau}+\sigma_{g, \mathcal{T}}\right)+\alpha^{1 / 2} \nabla \bar{u}_{\mathcal{T}}\right\|_{0, \Omega}=\min _{\boldsymbol{\tau} \in \mathcal{V}_{g}}\left\|\alpha^{-1 / 2} \boldsymbol{\tau}+\alpha^{1 / 2} \nabla \bar{u}_{\mathcal{T}}\right\|_{0, \Omega} .
$$

Next, based on the explicit approximation in (4.11), we define the explicit local a posteriori error indicator by

$$
\hat{\eta}_{\mathcal{V}, K}=\left\|\alpha^{-1 / 2} \hat{\boldsymbol{\sigma}}_{\mathcal{T}}+\alpha^{1 / 2} \nabla \bar{u}_{\mathcal{T}}\right\|_{0, K}
$$

for any $K \in \mathcal{T}$ and the explicit global a posteriori error estimator by

$$
\hat{\eta}_{\mathcal{V}}=\left(\sum_{K \in \mathcal{T}}\left(\hat{\eta}_{\mathcal{V}, K}\right)^{2}\right)^{1 / 2}=\left\|\alpha^{-1 / 2} \hat{\boldsymbol{\sigma}}_{\mathcal{T}}+\alpha^{1 / 2} \nabla \bar{u}_{\mathcal{T}}\right\|_{0, \Omega}
$$




\section{RELIABILITY AND EFFICIENCY BOUNDS FOR RECOVERY-BASED ERROR ESTIMATORS}

In this section, we establish reliability and efficiency bounds for both implicit and explicit estimators.

\section{A. Reliability}

Theorem 5.1. Assume that $\bar{u}_{\mathcal{T}}=u_{\mathcal{T}}$ is the linear finite element solution of (2.2). Then the estimator $\eta_{\mathcal{V}}$ defined in (4.15) satisfies the following global reliability bound:

$$
\|e\| \leq C\left(\eta_{\mathcal{V}}+\hat{H}_{f}+H_{g}+H_{g_{N}}\right),
$$

where $C$ is a constant independent of the ratio $\alpha_{\max } / \alpha_{\min }$

Proof. It follows from the orthogonality property of the finite element solution, integration by parts, (1.1), and the Cauchy-Schwarz inequality that

$$
\begin{aligned}
\|e\|^{2}= & a(e, e-I e)=\left(\alpha \nabla\left(u-u_{\mathcal{T}}\right), \nabla(e-I e)\right) \\
= & \left(\alpha \nabla u+\sigma_{\mathcal{T}}, \nabla(e-I e)\right)-\left(\sigma_{\mathcal{T}}+\alpha \nabla u_{\mathcal{T}}, \nabla(e-I e)\right) \\
\leq & \left(f-\nabla \cdot \sigma_{\mathcal{T}}, e-I e\right)+\left\langle g+\llbracket \boldsymbol{\sigma}_{\mathcal{T}} \cdot \mathbf{n} \rrbracket, e-I e\right\rangle_{\mathcal{S}} \\
& +\left\langle g_{N}-g_{N, \mathcal{T}}, e-I e\right\rangle_{\Gamma_{N}}+\eta_{\mathcal{V}}\|e-I e\|,
\end{aligned}
$$

which, combining with the fact that $\left.\nabla \cdot\left(\alpha(x) \nabla u_{\mathcal{T}}\right)\right|_{K}=0$ for all $K \in \mathcal{T},(3.12)$, (3.11), and the Cauchy-Schwarz inequality, implies

$$
\begin{aligned}
\|e\|^{2} \leq & (f, e-I e)-\sum_{K \in \mathcal{T}}\left(\nabla \cdot\left(\sigma_{\mathcal{T}}+\alpha \nabla u_{\mathcal{T}}\right), e-I e\right)_{K}+\left\langle g-g_{\mathcal{T}}, e-I e\right\rangle_{\mathcal{S}} \\
& +\left\langle g_{N}-g_{N, \mathcal{T}}, e-I e\right\rangle_{\Gamma_{N}}+C \eta_{\mathcal{V}}\|e\| \\
\leq & C\left(\hat{H}_{f}+H_{g}+H_{g_{N}}+\eta_{\mathcal{V}}\right)\|e\| \\
& +\left(\sum_{K \in \mathcal{T}} h_{K}^{2}\left\|\nabla \cdot\left(\alpha^{-1 / 2} \sigma_{\mathcal{T}}+\alpha^{1 / 2} \nabla u_{\mathcal{T}}\right)\right\|_{0, K}^{2}\right)^{1 / 2}\left(\sum_{K \in \mathcal{T}} h_{K}^{-2} \alpha_{K}\|e-I e\|_{0, K}^{2}\right)^{1 / 2}
\end{aligned}
$$

Using the inverse inequality and (2.3), we then have

$$
\|e\|^{2} \leq C\left(\hat{H}_{f}+H_{g}+H_{g_{N}}+\eta_{\mathcal{V}}\right)\|e\|+C \eta_{\mathcal{V}}\|e\|=C\left(\hat{H}_{f}+H_{g}+H_{g_{N}}+\eta_{\mathcal{V}}\right)\|e\|,
$$

which leads to (5.1). This completes the proof of the theorem.

Theorem 5.2. Under the same assumption of Theorem 5.1, the explicit estimator $\hat{\eta}_{\mathcal{V}}$ defined in (4.18) satisfies the following global reliability bound:

$$
\|e\| \leq C\left(\hat{\eta}_{\mathcal{V}}+\hat{H}_{f}+H_{g}+H_{g_{N}}\right)
$$

where $C$ is a constant independent of the ratio $\alpha_{\max } / \alpha_{\min }$. 
Proof. The reliability bound in (5.2) is an immediate consequence of (5.1) and the fact that

$$
\hat{\eta}_{\mathcal{V}} \geq \min _{\boldsymbol{\tau} \in \mathcal{V}_{g}}\left\|\alpha(x)^{-1 / 2} \boldsymbol{\tau}+\alpha(x)^{1 / 2} \nabla u_{\mathcal{T}}\right\|_{0, \Omega}=\eta_{\mathcal{V}}
$$

This completes the proof of the theorem.

\section{B. Efficiency}

Lemma 5.3. For any element $K \in \mathcal{T}$, the constant vector $\boldsymbol{\tau}$ on $K$ has the following representation in $\mathcal{V}$ :

$$
\boldsymbol{\tau}=\sum_{e \in \partial K} \tau_{e, K} \boldsymbol{\phi}_{e}(\mathbf{x})
$$

where $\tau_{e, K}=\left.\left(\left.\boldsymbol{\tau}\right|_{K} \cdot \mathbf{n}_{e}\right)\right|_{e}$ is the normal component of $\boldsymbol{\tau}$ on edge $e$

Proof. The lemma is a direct consequence of the fact, that $R T_{0}(K)$ contains the constant vector and the properties of the nodal basis functions (4.10).

Theorem 5.4. There exists a constant $C>0$ independent of $\alpha_{\max } / \alpha_{\min }$ such that

$$
\hat{\eta}_{\mathcal{V}, K}^{2} \leq C\left(\|e\|_{\omega_{K}}^{2}+\sum_{T \in \mathcal{T} \cap \omega_{K}} H_{f, K}^{2}+\sum_{e \in \partial K \cap \mathcal{E}_{N}} H_{g_{N}, e}^{2}+\sum_{e \in \partial K \cap \mathcal{E}_{\mathcal{S}}} H_{g, e}^{2}\right)
$$

where $\omega_{K}$ is the union of elements sharing a common edgelface with $K$, and that

$$
\eta_{\mathcal{V}} \leq \hat{\eta}_{\mathcal{V}} \leq C\left(\|e\|_{\Omega}+H_{f}+H_{g}+H_{g_{N}}\right)
$$

Proof. For any element $K \in \mathcal{T}$ and for any edge/face $e \in \partial K$, without loss of generality, assume that $\mathbf{n}_{e}$ is the outward unit vector normal to $\partial K$. Denote by $K_{e}$ the adjacent element with common edge/face $e$. Let $\boldsymbol{\tau}=-\alpha \nabla u_{\mathcal{T}}$, then, for any $\mathbf{x} \in K$, (4.11), (4.12), and (5.3) give

$$
\begin{aligned}
\hat{\boldsymbol{\sigma}}_{\mathcal{T}}-\boldsymbol{\tau}= & \sum_{e \in \mathcal{E}_{K}}\left(\hat{\sigma}_{e}-\tau_{e, K}\right) \boldsymbol{\phi}_{e}(\mathbf{x}) \\
= & \sum_{e \in \mathcal{E}_{K} \cap \mathcal{E}_{\Omega}}\left(1-\gamma_{e}\right)\left(\tau_{e, K_{e}}-\tau_{e, K}\right) \boldsymbol{\phi}_{e}(\mathbf{x})+\sum_{e \in \mathcal{E}_{K} \cap \mathcal{E}_{N}}\left(-g_{N, \mathcal{T}}-\tau_{e, K}\right) \boldsymbol{\phi}_{e}(\mathbf{x}) \\
& +\sum_{e \in \mathcal{E}_{K} \cap \mathcal{E}_{\mathcal{S}}}\left(1-\gamma_{e}\right)\left(\tau_{e, K_{e}}-\tau_{e, K}-g_{\mathcal{T}}\right) \boldsymbol{\phi}_{e}(\mathbf{x}) \\
= & \sum_{e \in \mathcal{E}_{K} \cap\left(\mathcal{E}_{\Omega} \cup \mathcal{E}_{\mathcal{S}}\right)}\left(1-\gamma_{e}\right) J_{e}\left(u_{\mathcal{T}}\right) \boldsymbol{\phi}_{e}(\mathbf{x})-\sum_{e \in \mathcal{E}_{K} \cap \mathcal{E}_{N}} J_{e}\left(u_{\mathcal{T}}\right) \boldsymbol{\phi}_{e}(\mathbf{x}) .
\end{aligned}
$$


Since $J_{e}\left(u_{\mathcal{T}}\right)$ is constant in $K$ and $\left\|\boldsymbol{\phi}_{e}(\mathbf{x})\right\|_{0, K}^{2} \leq C|K|$, it then follows from the triangle inequality and (4.13) that

$$
\begin{aligned}
\hat{\eta}_{\mathcal{V}, K}^{2} & =\left\|\alpha^{-1 / 2}\left(\hat{\boldsymbol{\sigma}}_{\mathcal{T}}-\boldsymbol{\tau}\right)\right\|_{0, K}^{2} \\
& \leq C \sum_{e \in \mathcal{E}_{K} \cap\left(\mathcal{E}_{\Omega} \cup \mathcal{E}_{\mathcal{S}}\right)} \frac{\left(1-\gamma_{e}\right)^{2}}{\alpha_{K}}\left\|J_{e}\left(u_{\mathcal{T}}\right) \boldsymbol{\phi}_{e}(\mathbf{x})\right\|_{0, K}^{2}+\sum_{e \in \mathcal{E}_{K} \cap \mathcal{E}_{N}} \frac{1}{\alpha_{K}}\left\|J_{e}\left(u_{\mathcal{T}}\right) \boldsymbol{\phi}_{e}(\mathbf{x})\right\|_{0, K}^{2} \\
& \leq C \sum_{e \in \mathcal{E}_{K} \cap\left(\mathcal{E}_{\Omega} \cup \mathcal{E}_{\mathcal{S}}\right)} \frac{1}{\alpha_{K}+\alpha_{K_{e}}}\left|J_{e}\left(u_{\mathcal{T}}\right)\right|^{2}\left\|\boldsymbol{\phi}_{e}(\mathbf{x})\right\|_{0, K}^{2}+\sum_{e \in \mathcal{E}_{K} \cap \mathcal{E}_{N}} \frac{1}{\alpha_{K}}\left|J_{e}\left(u_{\mathcal{T}}\right)\right|^{2}\left\|\boldsymbol{\phi}_{e}(\mathbf{x})\right\|_{0, K}^{2} \\
& \leq C \sum_{e \in \mathcal{E}_{K} \cap\left(\mathcal{E}_{\Omega} \cup \mathcal{E}_{\mathcal{S}} \cup \mathcal{E}_{N}\right)} \eta_{e}^{2},
\end{aligned}
$$

which, together with (3.18), implies (5.4).

The first inequality in the global efficiency bound (5.5) follows from (4.16). Summing up (5.4) over all $K \in \mathcal{T}$ proves the second one in (5.5).

\section{References}

1. L. Chen, M. J. Holst, and J. Xu, The finite element approximation of the nonlinear Poisson-Boltzmann equation, SIAM J Numer Anal 45 (2007), 2298-2320.

2. Z. Li and K. Ito, The immersed interface method - numerical solutions of PDEs involving interfaces and irregular domains, SIAM, 2006.

3. R. Verfürth, A review of a posteriori error estimation and adaptive mesh-refinement techniques, Wiley-Teubner, Stuttgart, Germany, 1996.

4. M. Ainsworth and J. T. Oden, A posteriori error estimation in finite element analysis, Wiley-Interscience, New York, 2000.

5. I. Babuska and T. Strouboulis, The finite element method and its reliability, Oxford Science Publication, New York, 2001.

6. C. Bernardi and R. Verfürth, Adaptive finite element methods for elliptic equations with non-smooth coefficients, Numer Math 85 (2000), 579-608.

7. M. Petzoldt, A posteriori error estimators for elliptic equations with discontinuous coefficients, Adv Comp Math 16 (2002), 47-75.

8. Z. Cai and S. Zhang, Recovery-based error estimator for interface problems: conforming linear elements, SIAM J Numer Anal 47 (2009), 2132-2156.

9. Z. Cai and S. Zhang, Recovery-based error estimators for interface problems: mixed and nonconforming finite elements, SIAM J Numer Anal 48 (2010), 30-52.

10. Z. Cai, X. Ye, and S. Zhang, Discontinuous Galerkin finite element methods for interface problems: a priori and a posteriori error estimations, to appear.

11. O. C. Zienkiewicz and J. Z. Zhu, A simple error estimator and adaptive procedure for practical engineering analysis, Int J Numer Methods Eng 24 (1987), 337-357.

12. C. Carstensen and S. Bartels, Each averaging technique yields reliable a posteriori error control in FEM on unstructure grids. Part I: low order conforming, nonconforming, and mixed FEM, Math Comp 71 (2002), 945-969.

13. J. S. Ovall, Two dangers to avoid when using gradient recovery methods for finite element error estimation and adaptivity, Max-Planck-Institute fur Mathematick in den Naturwissenschaften, Leipzig, Tech. Rep. no. 6, 2006. 
14. J. S. Ovall, Fixing a "bug" in recovery-type a posteriori error estimators, Max-Planck-Institute fur Mathematick in den Naturwissenschaften, Leipzig, Tech. Rep. no. 25, 2006.

15. P. G. Ciarlet, The finite element method for elliptic problems, North-Holland, Amsterdam, 1978.

16. C. Carstensen and R. Verfürth, Edge residuals dominate a posteriori error estimates for low order finite element methods, SIAM J Numer Anal 36 (1999), 1571-1587.

17. J. L. Lions and E. Magenes, Non-homogeneous boundary value problems and applications, Vol. I, Springer, New York, 1972.

18. F. Brezzi and M. Fortin, Mixed and hybrid finite element methods, Springer-Verlag, New York, 1991.

19. P. A. Raviart and I. M. Thomas, A mixed finite element method for second order elliptic problems, Lect. Notes Math. 606, Springer-Verlag, Berlin, New York, 1977, 292-315. 